\title{
Molecular Imaging: Principles and Practice
}

\author{
R. Weissleder, B.D. Ross, A. Rehemtulla, and S.S. Gambhir
}

Shelton, CT: People's Medical Publishing House-USA, 2010, 1357 pages, $\$ 189$

The field of molecular imaging has seen spectacular advances in chemistry, engineering, and biochemical applications in recent years. Comprehensive molecular imaging centers have been established all over the world, and new research workers, as well as students, have been drawn into this multidisciplinary field, creating a compelling need for a textbook to define it and outline the underlying science, techniques, and clinical applications. In an effort to meet this need, 167 leaders in molecular imaging have contributed the 76 chapters of this book, in which a large amount of information has been logically organized to provide the most authoritative and effective resource currently available, illustrated with molecular imaging examples.

This book is the first to cover all aspects of molecular imaging, including the various modalities and biomedical applications and the chemistry of imaging agent development and imaging instrument strategies.

The book is broken into 6 parts following general principles. Part I (molecular imaging technologies) summarizes the different imaging techniques, with 18 chapters dealing with PET/CT, PET/MRI, SPECT/CT, micro x-ray $\mathrm{CT}$, bioluminescent markers, optical imaging, fluorescence tomography, endomicroscopy, intravital microscopy, ultrasonography, and photoacoustic tomography. Part II (chemistry of molecular imaging) reviews chemical approaches to designing probes for different types of imaging methods. This part contains 19 chapters on the emerging field of nanomaterials, chemical biology, and probe design, as well as signal amplification strategies. It also includes the radiochemistry of PET and SPECT, antibodies and peptides, aptamers, ${ }^{13} \mathrm{C}$ MRI, click chemistry, and theranostics. Part III (molecular imaging in cell and molecular biology) contains 12 chapters dedicated to protein engineering, vectors, and pathways. System biology, phage display, gene therapy, tumor vasculature, hypoxia, and fluorescent readouts are discussed. Part IV (applications of molecular imaging) summarizes the advanced techniques of molecular imaging in different clinical disease entities. The 17 chapters are grouped into 4 sections: oncology, the cardiovascular system, the central nervous system, and autoimmune/ immunology. Part V (molecular imaging in drug evaluation) consists of 4 chapters dedicated to imaging in drug development and gene therapy, including clinical trials with PET and MRI. Part VI (other) provides 6 chapters on computation, bioinformatics, and modeling. Cost-effectiveness analysis and the regulatory and reimbursement process are also discussed.

There are few redundancies despite the many chapters and large amount of logical information. The clear, informative figures illustrate the concepts well and show many examples of molecular imaging. The tables, updated references, and index are useful and helpful. This book is the most authoritative and effective resource for imaging students and practitioners at all levels for the integration of molecular imaging into basic sciences and translational networks. I highly recommend it to trainees and practitioners in the fields of nuclear medicine and radiology and to imaging scientists and research workers using molecular imaging techniques in oncology, neurology, and cardiology.

\section{E. Edmund Kim}

M.D. Anderson Cancer Center

1515 Holcombe Blvd., Unit 1264

Houston, TX 77030

E-mail: ekim@di.mdacc.tmc.edu 